\title{
A Method of State-of-Charge Estimation for EV Power Lithium-ion Battery Using A Novel Adaptive Extended Kalman Filter
}

\author{
Zhicheng He, Ziming Yang, Xiangyu Cui and Eric Li
}

\begin{abstract}
Battery management system (BMS) is one of the
\end{abstract} key subsystems of electric vehicle, and the battery state-of -charge (SOC) is a crucial input for the calculations of energy and power. Therefore, SOC estimation is a significant task for BMS. In this paper, a new method for online estimating SOC is proposed, which combines a novel adaptive extended Kalman filter (AEKF) and a parameter identification algorithm based on adaptive recursive least squares (RLS). Specifically, according to the first order R-C network equivalent circuit model, the battery model parameters are identified online using the RLS with multiple forgetting factors. Based on the identified parameters, the novel AEKF is used to accurately estimate the battery SOC. The online identification of parameter tracks the varying model. At the same time, due to the novel AEKF algorithm to dynamically adjust the system noise parameter, excellent accuracy of the SOC real-time estimation is obtained. Experiments are conducted to evaluate the accuracy and robustness of the proposed SOC estimation method. The simulation test results indicate that under DST and UDDS conditions, the maximum absolute errors are less than 0.015 after filtering convergence. In addition, the maximum absolute error is less than 0.02 in the simulation of DST with current and voltage measurement noise, so is in DST with current offset sensor error. The tests indicate that the proposed method can accurately estimate battery SOC and has strong robustness.

Index term-State of Charge, Adaptive Extended Kalman Filter, Online Identification, Lithium-ion battery, Electric Vehicle

\section{I . INTRODUCTION}

s the performance of lithium-ion battery continues to
increase, lithium-ion battery is widely applied as energy storage device for electric vehicles [1]-[4]. SOC directly affects the calculation of the power and energy of the battery, so it is ${ }^{1}$ essential for EV energy management. The estimation accuracy, robustness, and anti-disturbance ability are important indicators for evaluating the performance of SOC estimation [5]-[7], thus SOC estimation approaches should focus on them.

The Coulomb counting [8] method has low computational complexity and is easy to implement. It can operate efficiently for a short period of time when the initial state is known. However, due to current measurement bias, after long-term operation of this method, the integration process will accumulate errors, which will lead to large estimation errors. SOC estimation approach based on looking up OCV-SOC table has been applied in some fields because it is easy to implement [9]. Unfortunately, this method requires the battery to stand still for a long time so that the chemical reaction inside the battery is completely calmed, thus it is not real-time. Scholars have successively adopted various online data-driven methods to battery SOC estimation, such as fuzzy logic [10], neural network [11], sliding mode observer [12], non-linear observers [13], support vector machine [14] and proportional-integral observer [15] and model-based estimators [16]-[22].

According to the sensor measurements (voltage and current), model-based estimators apply algorithms to infer the SOC. Among a variety of battery models, the extensively applied models include neural network model [16] [17], equivalent circuit models (ECM) [18]-[21], electrochemical models [22] [23]. ECM-based estimation methods have been widely studied for the good accuracy and moderate practical burden. Kalman filter is a common algorithm for estimating the internal states of

Zhicheng He and Ziming Yang are with State Key Laboratory of Advanced Design and Manufacturing for Vehicle Body, Hunan University, 410082 Changsha, P. R. China.

Xiangyu Cui is with Key Laboratory of Electrochemical Energy Storage and Energy Conversion of Hainan Province, College of Physics and Electronic Engineering of Hainan Normal University, 571158 Haikou, P. R. China.

Eric Li is with School of Computing, Engineering \& Digital Technologies, Teesside University, Middlesbrough, UK. 
a dynamic system, and it has a good application in many fields [24]-[28]. The Kalman filter-based methods rely on the accuracy of the ECMs for the battery, and SOC estimation is affected by the varying battery parameters. Therefore, SOC estimation is inseparable from the identification of system parameters. Some methods [29] [30] regard the parameters as a function of SOC, and identify the model parameters offline through experiments of various working conditions, but this method need high development cost and long development cycle.

SOC estimation and parameter identification are often performed simultaneously, and methods based on EKF and RLS have been extensively utilized [31]-[35]. In [31], SOC is estimated using KF and the errors are analyzed, then RLS is utilized for parameter correction. Reference [32] proposed a co-estimation method combining EKF and bias compensation RLS, reducing the estimation errors causing by measurement bias. Wei [33] proposed a SOC and capacity dual estimator, and constructed a complete online model identification and SOC estimation framework by using the vector-type recursive least squares (VRLS) and Kalman filter. Reference [34] proposed an online estimator based on VFFRLS and CKF, and it indicated that the CKF outperforms EKF in SOC estimation of lithium-ion battery. Considering the SOC constraints, the estimator in [35] uses RLS and a improve EKF to estimate SOC of vanadium redox battery, enhancing the accuracy, convergence speed and robustness. These methods strictly require the accuracy of the model that describe the electrical characteristics of the battery system.

For EKF-based state estimation methods, determining appropriate system noise parameters is critical for EKF-based SOC estimation methods. The noise parameters include process noise covariance and measurement noise covariance, which is used to characterize the statistical characteristics of the noise in the state equation and measurement equation, and represented by $Q$ and $R$ respectively. As for ECM-based SOC estimation, these noise parameters are related to model uncertainty and measurement bias. The model of the battery is not absolute, and the model uncertainties from ECMs are inevitable, which is evidenced by [36]-[38]. The ECMs can simulate the electrical behavior of the battery system to some extent, but cannot represent the actual physical characteristics. Generally, uncertainties inevitably exist in ECMs, and the accuracy of the model always positively related to its complexity [39]-[41].
The measurement bias come from external disturbance and sensor deviations, and directly affect calculation accuracy. Reference [42] points out that suitable Kalman gains can reduce the influence of measurement bias, while the Kalman gains are affected by the coordination of process covariance and measurement covariance. To some extent, these uncertainties of model and measurement can be regarded as Gaussian white noise in EKF-based methods. Therefore, it is a significant task to find suitable noise parameters (exactly, the matching of process covariance and measurement covariance).

Inappropriate noise covariance will reduce estimation accuracy, and even lead to filter divergence. Unfortunately, noise parameters are unknown and difficult to determine. In order to solve this problem, many Kalman filter-based adaptive estimators have been proposed. The different adaptive filtering methods are classified into four categories in [43]: maximum likelihood, Bayesian, covariance matching and correlation. These approaches have been applied in various fields. Reference [44] utilizes FFRLS and IRVM-EKF to jointly estimate the model parameter and SOC, and a measurement model is used to reduce the influence of model uncertainty and measurement bias, while the noise parameter is only adjusted qualitatively. Xiong, et al. in [45] [46] applied covariance matching to calculate noise covariances of EKF and the SOC estimation method achieved quite high accuracy. The method proposed in [47] applies RLS and adaptive dual EKF to reduce the impact of model inaccuracy and current measurement error, and accurate estimation result and good robustness are achieved. Although the approaches mentioned above have achieved good results, there are still some issues. The second filter and the calculation of the system noise covariance matrices lengthens the algorithm flow, and the accuracy depends on the predetermination of the size of the innovation sequence sets and the adaptive factors.

In this paper, a new method for SOC online estimation is proposed. The approach consists of a novel adaptive extended Kalman filter and vector-type recursive least squares (VRLS). Specifically, the VRLS algorithm using multiple forgetting factors is used to identify the ECM parameters changing with different rates. A new adaptive extended Kalman filter directly adjusting the priori error covariance matrix is used to estimate the states, which can dynamically track the system noise. Moreover, the adaptive algorithm guarantees the positive definiteness of covariance, thus avoiding filtering divergence 
causing by non-positive definiteness. What's more, the adaptive algorithm flow is the simple because it directly adjusts the priori error covariance instead of the noise covariances. To verify its estimation accuracy and capacity of resisting disturbance, the proposed method was operated not only in classic DST and UDDS test, but also in DST with gaussian noise and current offset. The test results indicate the high estimation accuracy and strong robustness of the method. We hope that this study can provide some reference for SOC estimation in BMS. We also wish the application of the novel AEKF in state estimation of similar systems by scholars.

\section{BATTERY MODEL AND IDENTIFICATION OF PARAMETERS}

\section{A. RC Network-Based Battery Model}

In consideration of accuracy, parameter identification effort and the computational complexity, the first-order RC network-based circuit model is chosen to describe the electrical characteristics of the lithium-ion battery system, which is shown in Fig. 1. R0 stands for the ohmic resistance of the battery. The resistor capacitor (RC) network is used to describe the transient dynamics of the battery, which consists of polarization capacitance $(\mathrm{Cp})$ and polarization resistance $(\mathrm{Rp})$. Uoc is the open circuit voltage; IL represents the load current, the control input that can be measured by sensor; Ut represents the terminal voltage, which also can be measured by sensor; Up denotes the polarization voltage over the $\mathrm{RC}$ network.

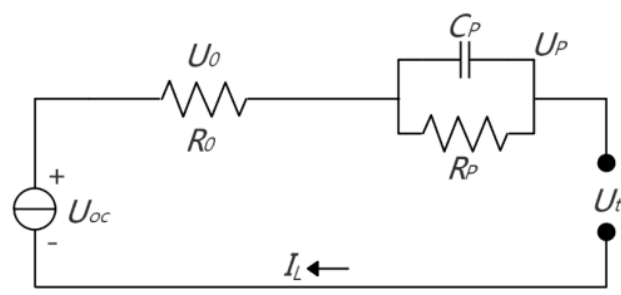

Fig. 1. First-order RC model of battery.

\section{B. Identification of Model Parameters}

The electrical behavior of the used ECM is generalized as the following state-space equations:

$I_{L}=C_{p} \frac{d U_{p}}{d t}+\frac{U_{p}}{R_{p}}$

$U_{o c}=U_{t}+U_{p}+I_{L} R_{0}$

Projecting (1) to discrete time domain yields (3):

$U_{p}(t)=e^{-\frac{T_{c}}{R p C_{p}}} U_{p}\left(t-T_{c}\right)+\left(1-e^{-\frac{T_{c}}{R_{p} C_{p}}}\right) R_{p} I_{L}\left(t-T_{c}\right)$

By substituting (3) into (2), the terminal voltage can be expressed as:

$U_{t}(t)=U_{o c}(t)-\theta_{1} U_{o c}\left(t-T_{c}\right)+\theta_{1} U_{t}\left(t-T_{c}\right)+\theta_{2} I_{L}(t)+$ $\theta_{3} I_{L}\left(t-T_{c}\right)$

where

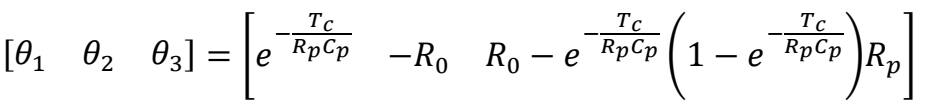
and $T_{c}$ is the sampling time interval of parameter estimator, which is set to $4 \mathrm{~s}$ in this study. According to (4), subtracting $U_{t}\left(t-T_{c}\right)$ from $U_{t}(t)$, the following regression equation can be organized:

$\Delta U_{t}(t)=h(t) \theta^{T}(t)+\xi(t)$

where

$h(t)=\left[\begin{array}{lll}\Delta U_{t}\left(t-T_{c}\right) & \Delta I_{t}(t) \quad \Delta I_{L}\left(t-T_{c}\right)\end{array}\right]$

$\theta(t)=\left[\begin{array}{lll}\theta_{1} & \theta_{2} & \theta_{3}\end{array}\right]$

$\xi(t)=\Delta\left[U_{o c}(t)-\theta_{1} U_{o c}\left(t-T_{c}\right)\right]$

and $\Delta U_{t}(t)$ is calculated by the terminal voltages of the two adjacent time steps, that is:

$\Delta U_{t}(t)=U_{t}(t)-U_{t}\left(t-T_{c}\right)$

In the similar way, the items in (7) and (9) are calculated with the following equations:

$\Delta I_{t}(t)=I_{t}(t)-I_{t}\left(t-T_{c}\right)$

$\Delta\left[U_{o c}(t)-\theta_{1} U_{o c}\left(t-T_{c}\right)\right]=\left[U_{o c}(t)-\theta_{1} U_{o c}\left(t-T_{c}\right)\right]$

$-\left[U_{o c}\left(t-T_{c}\right)-\theta_{1} U_{o c}\left(t-2 T_{c}\right)\right]$

In microscopic time, the change of OCV is small enough to be negligible, and it is reasonable to ignore the residual term $\xi(t)$.

The regression (6) can be solved by the RLS. In order to track the parameters with different rates of change, the vector-type forgetting recursive least squares (VRLS) is used, which uses multiple forgetting factors. In addition, the factors change over time. A small factor is used for tracking in the early stage while a big factor is used for stability in the later stage. The forgetting factors are calculated by the following formula:

$\lambda_{\theta_{i}}=\lambda_{\max _{i}}+\left(\lambda_{\min _{i}}-\lambda_{\max _{i}}\right) \cdot 2^{-\frac{t}{\tau_{i}}}$

where $i=1,2,3 . \lambda_{\theta_{1}}, \lambda_{\theta_{2}}, \lambda_{\theta_{3}}$ are the forgetting factors used for identifying $\theta_{1}, \theta_{2}, \theta_{3}, \lambda_{\max _{i}}$ and $\lambda_{\min _{i}}$ is the maximum value and minimum value of the forgetting factor respectively, $\tau_{i}$ is the time constant. The values of these coefficients used are listed in TABLE I. The algorithm flow of VRLS is shown in TABLE II. After the regression (6) is solved, the model parameters can be calculated by the following formula:

$\left[\begin{array}{lll}R_{0} & R_{p} & C_{p}\end{array}\right]=\left[\begin{array}{lll}-\theta_{2} & \frac{\theta_{1} \theta_{2}+\theta_{3}}{\theta_{1}-1} & \frac{\left(1-\theta_{1}\right) T_{c}}{\left(\theta_{1} \theta_{2}+\theta_{3}\right) \ln \theta_{1}}\end{array}\right]$

TABLE I

THE VALUES OF COEFFICIENTS TO CALCULATE FORGETTING FACTORS

\begin{tabular}{rrrl}
\hline \hline$i$ & $\lambda_{\max _{i}}$ & $\lambda_{\min _{i}}$ & $\tau_{i}$ \\
\hline 1 & 0.99 & 0.97 & 750 \\
2 & 0.98 & 0.96 & 750
\end{tabular}


3

0.99

0.97

750

TABLE II

THE ALGORITHM FLOW OF VRLS

Step 1. Initialization1

Parameter:

$$
\hat{\theta}_{0}^{+}=E\left(\theta_{0}\right)
$$

Posteriori covariance:

$$
P_{0}^{+}=E\left[\left(\theta_{0}-\hat{\theta}_{0 \mid 0}\right)\left(\theta_{0}-\hat{\theta}_{0 \mid 0}\right)^{T}\right]
$$

Step 2. Priori parameter and covariance update

Priori parameter: $\hat{\theta}_{k}^{-}=\hat{\theta}_{k-1}^{+}$

Priori covariance:

$$
P_{k}^{-}=P_{k-1}^{+}
$$

Step 3. Gain update

Gain:

$$
G_{k}=P_{k}^{-}\left(h_{k}\right)^{T}\left[1+h_{k} P_{k}^{-}\left(h_{k}\right)^{T}\right]^{-1}
$$

Step 4. Posteriori parameter and covariance update

$$
\begin{aligned}
& \text { Posteriori parameter: } \\
& \qquad \hat{\theta}_{k}^{+}=\hat{\theta}_{k}^{-}+G_{k}\left[\Delta U_{t, k}-h_{k}\left(\hat{\theta}_{k}^{-}\right)^{T}\right]
\end{aligned}
$$

Posteriori covariance:

$$
P_{k}^{+}=\Lambda^{-1}\left(I-G_{k} h_{k}\right) P_{k}^{-} \Lambda^{-1}
$$

Where

$$
\Lambda=\operatorname{diag}\left(\left[\begin{array}{lll}
\sqrt{\lambda_{\theta_{1}}} & \sqrt{\lambda_{\theta_{2}}} & \sqrt{\lambda_{\theta_{3}}}
\end{array}\right]\right)
$$

\section{SOC ESTIMATION ALGORITHM}

\section{A. State-Estimation Model}

According to Coulomb counting method, the recursive formula of SOC on discrete time domain can be written as:

$S O C_{k}=S O C_{k-1}-\frac{\eta_{k} T_{S} I_{L}}{q_{k}}$

where $\eta_{k}$ is Coulomb coefficient, $T_{s}$ is the timescale of SOC estimator, which is set to $1 \mathrm{~s}$ in this study, and $q_{k}$ is the battery capacity. Combining (3) and (23) generates the battery system state-space equations:

$$
\begin{aligned}
& x_{k}=\left[\begin{array}{c}
U_{p, k} \\
S O C_{k}
\end{array}\right]=\left[\begin{array}{cc}
\exp \left(-\frac{T_{c}}{R_{p} C_{p}}\right) & 0 \\
0 & 1
\end{array}\right]\left[\begin{array}{c}
U_{p, k-1} \\
S O C_{k-1}
\end{array}\right]+ \\
& {\left[\begin{array}{c}
\left(1-\exp \left(-\frac{T_{c}}{R_{p} C_{p}}\right)\right) R_{p} \\
-\frac{\eta_{k} T_{S}}{q_{k}}
\end{array}\right] I_{L, k-1}+w_{k}} \\
& z_{k}=U_{t, k}=\left[\begin{array}{ll}
-1 & 0
\end{array}\right]\left[\begin{array}{c}
U_{p, k} \\
S O C_{k}
\end{array}\right]+U_{O C, k}+\left(-R_{0}\right) I_{L, k}+v_{k}
\end{aligned}
$$

The OCV is considered as a function of SOC, which is expressed as:

$U_{O C}=\varphi(S O C)=\sum_{i=0}^{n_{f}} a_{i} S O C^{i}$ where $a_{i}$ is obtained by polynomial fitting. The fitted curve is shown in Fig. 2, which is obtained by an 8th degree polynomial curve fitting. Once the state equation (24) and the observation equation (25) are determined, the SOC can be estimated based on the EKF framework.

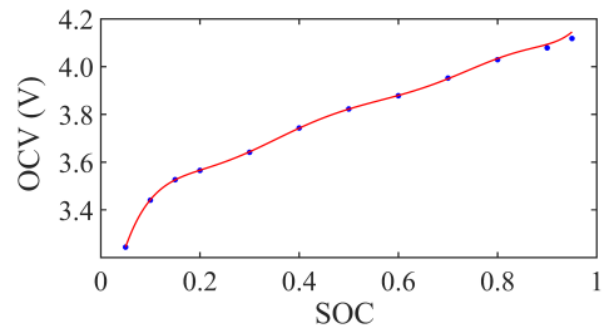

Fig. 2. Polynomial curve-fitted SOC-OCV correlation.

\section{B. New Adaptive Extended Kalman Filter}

Discrete state-space of $n$-dimensional non-linear system can be represented by the following equations:

$$
\begin{aligned}
& x_{k}=f\left(x_{k-1}, u_{k-1}\right)+w_{k}, k=0,1, \ldots \\
& z_{k}=g\left(x_{k}, u_{k}\right)+v_{k}, k=0,1, \ldots
\end{aligned}
$$

According to the estimate state $\hat{x}_{k-1 \mid k-1}$ of previous instant $k-1$, measurement $z_{k}$ and control input $u_{k-1}$ and $u_{k}$, extended Kalman filter output the estimate state $\hat{x}_{k \mid k}$ of instant $k$. $w_{k}$ and $v_{k}$ is the process noise and measurement noise. TABLE III presents the algorithm flow of standard extended Kalman filter (SEKF):

TABLE III

\section{FLOWCHART OF SEKF ALGORITHM}

Step 1. Initialization

(1) Initialize the state:

$$
\hat{x}_{0 \mid 0}=E\left(x_{0}\right)
$$

(2) Initialize the posterior error covariance:

$$
P_{0 \mid 0}=E\left[\left(x_{0}-\hat{x}_{0 \mid 0}\right)\left(x_{0}-\hat{x}_{0 \mid 0}\right)^{T}\right]
$$

Step 2. Prediction update

(1) Predict the state:

$$
\hat{x}_{k \mid k-1}=f\left(\hat{x}_{k-1 \mid k-1}, u_{k-1}\right)
$$

(2) Calculate the priori covariance:

$$
P_{k \mid k-1}=A_{k} P_{k-1 \mid k-1} A_{k}^{T}+Q_{k}
$$

where $A_{k}=\left.\frac{\partial f}{\partial\left(x^{T}\right)}\right|_{x_{k}=\hat{x}_{k-1 \mid k-1}}$

Step 3. Correction update

(1) Calculate the Kalman gain:

$$
K_{k}=P_{k \mid k-1} C_{k}^{T}\left[C_{k} P_{k \mid k-1} C_{k}^{T}+\left(R_{k}\right)^{-1}\right.
$$

(2) Estimate the state:

$$
\hat{x}_{k \mid k}=\hat{x}_{k \mid k-1}+K_{k}\left[z_{k}-g\left(\hat{x}_{k \mid k-1}, u_{k}\right)\right]
$$

(3) Calculate the posterior covariance:

$$
P_{k \mid k}=P_{k \mid k-1}-K_{k} C_{k} P_{k \mid k-1}
$$

where $C_{k}=\left.\frac{\partial g}{\partial\left(x^{T}\right)}\right|_{x_{k}=\hat{x}_{k \mid k}}$ 
From (24), (25) and (26), the state-space equations of lithium-ion battery system can be rewritten as:

$x_{k}=f\left(x_{k-1}, u_{k-1}\right)+w_{k}=A_{k} x_{k-1}+B_{k} I_{L, k-1}+w_{k}$

$z_{k}=g\left(x_{k}, u_{k}\right)+v_{k}=U_{O C, k}-U_{p, k}+\left(-R_{0}\right) I_{L, k}+v_{k}$

where $x_{k}=\left[\begin{array}{c}U_{p, k} \\ S O C_{k}\end{array}\right], z_{k}=U_{t, k}, A_{k}=\left[\begin{array}{cc}\exp \left(-\frac{T_{c}}{R_{p} C_{p}}\right) & 0 \\ 0 & 1\end{array}\right]$,

$B_{k}=\left[\begin{array}{c}\left(1-\exp \left(-\frac{T_{c}}{R_{p} C_{p}}\right)\right) R_{p} \\ -\frac{\eta_{k} T_{s}}{q_{k}}\end{array}\right]$. The corresponding matrices of

$C_{k}$ and $D_{k}$ in SEKF are expressed as:

$C_{k}=\left[\begin{array}{ll}-1 & \left.\frac{\partial U_{O C}}{\partial S O C}\right|_{S O C=\widehat{S O C}_{k \mid k-1}}\end{array}\right], D_{k}=-R_{0}$.

The different adaptive filtering approaches are classified into four categories in [43]: maximum likelihood, Bayesian, covariance matching and correlation. In this paper, we applied an adaptive extended Kalman filter that seeks an adaptive priori error covariance $P_{k \mid k-1}$. In an adaptive estimator, the innovation sequence is a key piece of statistics utilized to update the adaptive parameter, which is defined as:

$e_{k}=z_{k}-g\left(\hat{x}_{k \mid k-1}, u_{k}\right)$

The set of historical innovation sequence set that from instant $j_{0}$ to $k-1$ is expressed as:

$\varepsilon_{k}=\left\{e_{j_{0}}, e_{j_{0}+1}, \ldots, e_{k-1}\right\}$

The lithium-ion battery system parameters are slowly changing during the operation of the system. It has been proved in [48] that the priori error covariance $P_{k \mid k-1}$ is convergent if the system parameters gradually and slowly change. In fact, it is unrealistic for the approximation that the steady $P_{k \mid k-1}$ maintaining a constant in the practical process, which may reduce the filtering effect and even cause filtering divergence. However, this approximation is asymptotically efficient and can be used to explore a suboptimal but explicit way to estimate $P_{k \mid k-1}$ in real time. Similar assumptions and approximations can be seen in [43] [49] [50]. Let $\hat{P}_{k \mid k-1}$ represents the estimation of priori error covariance, and $p\left(\varepsilon_{k} \mid \hat{P}_{k \mid k-1}\right)$ represents the probability density function of the set $\varepsilon_{k}$ conditioned on $\hat{P}_{k \mid k-1}$, then based on maximum likelihood method, the maximum likelihood function can be written as:

$L\left(\hat{P}_{k \mid k-1}\right)=\ln p\left(\varepsilon_{k} \mid \hat{P}_{k \mid k-1}\right)=\ln \prod_{j=j_{0}}^{k-1} p\left(\mathrm{e}_{j} \mid \hat{P}_{k \mid k-1}\right)=$ $\sum_{j=j_{0}}^{k-1} \ln p\left(\mathrm{e}_{j} \mid \hat{P}_{k \mid k-1}\right)$

in which the probability density function of the sequence $e_{k}$ conditioned on $\hat{P}_{k \mid k-1}$ can be formulated as:

$$
\begin{aligned}
& p\left(\mathrm{e}_{j} \mid \hat{P}_{k \mid k-1}\right)=p\left(\mathrm{e}_{j} \mid \hat{P}_{j \mid j-1}\right) \\
& =\frac{1}{\sqrt{(2 \pi)^{m}\left|C_{e_{j}}\right|}} \exp \left(-\frac{1}{2} e_{j}^{T} C_{e_{j}}^{-1} e_{j}\right)
\end{aligned}
$$

where $m$ is the number of measurements; $C_{e_{k}}$ denotes the covariance matrix through innovation $e_{k} ;|\cdot|$ is the determinant operator. Taking the logarithm of (41), the following equation can be obtained:

$\ln p\left(\mathrm{e}_{j} \mid \hat{P}_{k \mid k-1}\right)=-\frac{1}{2}\left[m * \ln (2 \pi)+\ln \left|C_{e_{j}}\right|+e_{j}^{T} C_{e_{j}}^{-1} e_{j}\right]$

The maximum likelihood criterion for maximizing $p$ is organized into the following equation:

$M_{k}=\sum_{j=j_{0}}^{k-1} \ln \left|C_{e_{j}}\right|+\sum_{j=j_{0}}^{k-1} e_{j}^{T} C_{e_{j}}^{-1} e_{j}=\min$

In order to obtain the minimum of $M_{k}$, let the derivative of the maximum likelihood function $M_{k}$ equal zero:

$\sum_{j=j_{0}}^{k-1}\left[\operatorname{tr}\left(C_{e_{j}}^{-1} \frac{\partial C_{e_{j}}}{\hat{P}_{k \mid k-1}}\right)-e_{j} C_{e_{j}}^{-1} \frac{\partial C_{e_{j}}}{\hat{P}_{k \mid k-1}} C_{e_{j}}^{-1} e_{j}^{T}\right]=0$

where $\operatorname{tr}$ is the trace operator. Since $C_{e_{j}}=C_{j} P_{k \mid k-1} C_{j}^{T}+R$, (46) can be converted into:

$$
\begin{aligned}
& \sum_{j=j_{0}}^{k-1}\left[\operatorname{tr}\left(C_{e_{j}}^{-1} C_{j} \frac{\partial P_{j \mid j-1}}{\hat{P}_{k \mid k-1}} C_{j}^{T}\right)-e_{j} C_{e_{j}}^{-1} C_{j} \frac{\partial P_{j \mid j-1}}{\hat{P}_{k \mid k-1}} C_{j}^{T} C_{e_{j}}^{-1} e_{j}^{T}\right] \\
& =0
\end{aligned}
$$

As the EKF algorithm operating, the error of the estimated state of the state quickly approaches zero. Therefore, the priori error covariance $P_{k \mid k-1}$ tends to be convergent. ${ }^{58}$ Using the approximation that $P_{k \mid k-1}$ tends to be constant, $\frac{\partial P_{j \mid j-1}}{\hat{P}_{k \mid k-1}}$ and $\frac{\partial \hat{P}_{k \mid k-1}}{\hat{P}_{k \mid k-1}}$ are equal and they approximately equal to identity matrix. Then (47) reduces to

$\sum_{j=j_{0}}^{k-1}\left[\operatorname{tr}\left(C_{e_{j}}^{-1} C_{j} C_{j}^{T}\right)-e_{j} C_{e_{j}}^{-1} C_{j} C_{j}^{T} C_{e_{j}}^{-1} e_{j}^{T}\right]=0$

The following formula represents the necessary and sufficient condition for (48):

$\operatorname{tr}\left(C_{e_{j}}^{-1} C_{j} C_{j}^{T}\right)-e_{j} C_{e_{j}}^{-1} C_{j} C_{j}^{T} C_{e_{j}}^{-1} e_{j}^{T}=0$

From (39) and (40) it can be known that $C_{e_{j}}$ and $e_{j}$ are $1 \times 1$ matrix (or scalar), thus (49) can be organized into

$\left(C_{e_{j}}^{-1}-C_{e_{j}}^{-1} e_{j} e_{j}^{T} C_{e_{j}}^{-1}\right) C_{j} C_{j}^{T}=0$

Pre-multiply (50) by $C_{j}^{T}$, and post-multiply (50) by the inverse of $C_{j}^{T}$ (or the general inverse), 
$C_{j}^{T}\left(C_{e_{j}}^{-1}-C_{e_{j}}^{-1} e_{j} e_{j}^{T} C_{e_{j}}^{-1}\right) C_{j}=0$

Pre- and post-multiplying (51) by $P_{j \mid j-1}$ obtains:

$P_{j \mid j-1} C_{j}^{T}\left(C_{e_{j}}^{-1}-C_{e_{j}}^{-1} e_{j} e_{j}^{T} C_{e_{j}}^{-1}\right) C_{j} P_{j \mid j-1}=0$

Since $C_{e_{j}}=C_{j} P_{j \mid j-1} C_{j}^{T}+R, C_{e_{j}}$ is $1 \times 1$ matrix and $P_{j \mid j-1}$ is symmetric positive definite, namely $\left(C_{e_{j}}^{-1}\right)^{T}=C_{e_{j}}^{-1}$, $P_{j \mid j-1}^{T}=P_{j \mid j-1}$, substituting ( 34) into (52) obtains the following equation:

$K_{j} C_{j} P_{j \mid j-1}-K_{j} e_{j} e_{j}^{T} K_{j}^{T}=0$

Let

$\Delta \hat{x}_{j}=\hat{x}_{j}-\hat{x}_{j \mid j-1}=K_{j} e_{j}$

(36) and (54) at instant $j$ can be respectively written as $P_{j \mid j}=P_{j \mid j-1}-K_{j} C_{j} P_{j \mid j-1}, \Delta \hat{x}_{j}=K_{j} e_{j}$. Substituting them into (53), (53) can be converted into:

$P_{j \mid j-1}-P_{j \mid j}-\Delta \hat{x}_{j} \Delta \hat{x}_{j}^{T}=0$

Then accumulating (55) from instant $j_{0}$ to $k-1$ forms

$\sum_{j=j_{0}}^{k-1}\left(P_{j \mid j-1}-P_{j \mid j}-\Delta \hat{x}_{j} \Delta \hat{x}_{j}^{T}\right)=0$

and further

$\sum_{j=j_{0}}^{k-1} P_{j \mid j-1}=\sum_{j=j_{0}}^{k-1}\left(P_{j \mid j}+\Delta \hat{x}_{j} \Delta \hat{x}_{j}^{T}\right)$

With the assumed approximation $P_{k \mid k-1}=P_{j \mid j-1}, j<k$, $P_{k \mid k-1}$ could be approximately calculated by averaging all the $P_{j \mid j-1}$ from instant $j_{0}$ to $k-1$, namely the estimated covariance $P_{k \mid k-1}$ can be obtained by:

$\hat{P}_{k \mid k-1}=\frac{1}{k-j_{0}} \sum_{j=j_{0}}^{k-1} P_{j \mid j-1}=\frac{1}{k-j_{0}} \sum_{j=j_{0}}^{k-1}\left(P_{j \mid j}+\Delta \hat{x}_{j} \Delta \hat{x}_{j}^{T}\right)$

Covariance $\hat{P}_{k-1 \mid k-2}$ is calculated by the similar way:

$\widehat{P}_{k-1 \mid k-2}=\frac{1}{k-1-j_{0}} \sum_{j=j_{0}}^{k-2} P_{j \mid j-1}=\frac{1}{k-1-j_{0}} \sum_{j=j_{0}}^{k-2}\left(P_{j \mid j}+\Delta \hat{x}_{j} \Delta \hat{x}_{j}^{T}\right)$

Then with (58) and (59), the recursive formula of $\hat{P}_{k \mid k-1}$ can be obtained by follows:

$\hat{P}_{k \mid k-1}=\frac{k-1-j_{0}}{k-j_{0}} \hat{P}_{k-1 \mid k-2}+$

$\frac{1}{k-j_{0}}\left(P_{k-1 \mid k-1}+\Delta \hat{x}_{k-1} \Delta \hat{x}_{k-1}^{T}\right)$

Substituting (36) into (60) obtains:

$\hat{P}_{k \mid k-1}=\widehat{P}_{k-1 \mid k-2}+\frac{1}{k-j_{0}}\left(\Delta \hat{x}_{k-1} \Delta \hat{x}_{k-1}^{T}-K_{k-1} C_{k-1} \hat{P}_{k-1 \mid k-2}\right)$
The derivation of the recursive formula of the estimated priori error covariance $\hat{P}_{k \mid k-1}$ is completed. The algorithm flow of the new adaptive extended Kalman filter consists of (29), (30), (31), (61), (34) and (35). This algorithm adjusts priori error covariance $P_{k \mid k-1}$ with the feedback information $\Delta \hat{x}_{k}$, implicitly estimating the statistical characteristics of process noise. The new AEKF adjusts the matching of matrices of process noise covariance and measurement noise covariance, then suitable Kalman gain is obtained. It should be noted that before applying the adaptive algorithm $\left(k \leq k_{0}\right)$, the standard extended Kalman filter is used to help filtering converge, which uses an inaccurate or nominal noise covariances $Q$ and $R$. The boundary point of roughly filtering convergence is that the innovation is small enough and stable. The algorithm flow of the novel adaptive extended Kalman filter that tracks priori error covariance is shown in TABLE IV.

TABLE IV

ALGORITHM FLOWCHART OF THE NOVEL AEKF

Step 1. Initialization

Initialize the state:

$$
\hat{x}_{0 \mid 0}=E\left(x_{0}\right)
$$

Initialize the posterior error covariance:

$$
P_{0 \mid 0}=E\left[\left(x_{0}-\hat{x}_{0 \mid 0}\right)\left(x_{0}-\hat{x}_{0 \mid 0}\right)^{T}\right]
$$

Step 2. State prediction

Predict the state:

$$
\hat{x}_{k \mid k-1}=f\left(\hat{x}_{k-1}, u_{k-1}\right)
$$

Calculate the priori error covariance:

$k \leq k_{0}$ (instant before filtering convergence),

$$
P_{k \mid k-1}=A_{k} P_{k-1 \mid k-1} A_{k}^{T}+Q_{k}
$$

$k>k_{0}$ (instant after filtering convergence),

$$
P_{k \mid k-1}=P_{k-1 \mid k-2}+\frac{\left(\Delta \hat{x}_{k-1} \Delta \hat{x}_{k-1}^{T}-K_{k-1} C_{k-1} P_{k-1 \mid k-2}\right)}{\left(k-k_{0}\right)}
$$

Step 3. Filtering correction

Calculate Kalman gain:

$$
K_{k}=P_{k \mid k-1} C_{k}^{T}\left[C_{k} P_{k \mid k-1} C_{k}^{T}+R_{k}\right]^{-1}
$$

Estimate the state:

$$
\hat{x}_{k \mid k}=\hat{x}_{k \mid k-1}+K_{k}\left[z_{k}-g\left(\hat{x}_{k \mid k-1}, u_{k}\right)\right]
$$

Calculate the posterior error covariance (only when $k \leq k_{0}$ ):

$$
P_{k \mid k}=P_{k \mid k-1}-K_{k} C_{k} P_{k \mid k-1}
$$

Remark. The derivation of this adaptive algorithm is suboptimal, because it is based on the assumption that the priori 
error covariances of the SOC estimator are similar at steady state. Optimal filters perform well when the accurate noise parameters are known. In the specific application, optimal filtering cannot be achieved as the accurate noise parameters for the battery system (24) and (25) are difficult to obtain, this algorithm can be considered as an alternative method.

\section{Algorithm Framework of SOC Estimation}

The proposed SOC estimation method consists of VRLS-based model parameter identification and a state estimation algorithm based on a novel adaptive extended Kalman filter. The framework of the proposed approach is shown in Fig. 3.

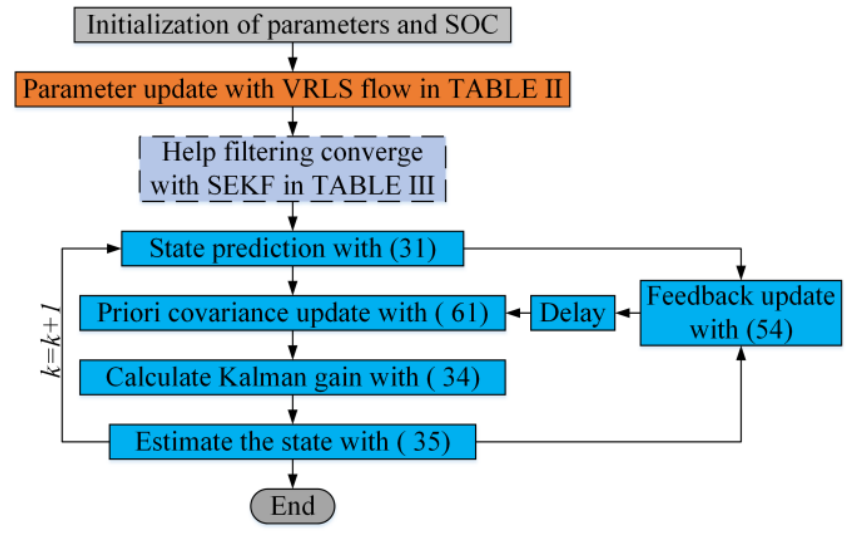

Fig. 3. Framework of the proposed adaptive SOC estimation algorithm.

\section{EXPERIMENT AND SIMULATION}

Experiments and simulations were set up to verify the accuracy and robust of the proposed SOC estimation method. Two typical tests are applied to evaluate the proposed method, including Dynamic Stress Test (DST) and Urban Dynamometer Driving Schedule (UDDS). The load current and the terminal voltage were obtained through bench experiments, as well as the reference SOC. The algorithm verification was operated in Matlab Simulink environment.

\section{A. Experimental Configuration}

A test bench shown in Fig. 4 was built on our own for experiments, which consists of battery cell, a device for battery charging/discharging (NBT BTS-5V300A), a thermal chamber for temperature control and a monitoring platform. The tested battery was made in Wanxiang Group, and its model number is WX35A. This test bench can perform test simulation of typical automotive conditions. The battery cell was charged/discharged in the thermal chamber with the NBT BTS-5V300A, and the temperature in the thermal chamber was kept around $25^{\circ} \mathrm{C}$.The monitoring platform load the current profile to the NBT BTS-5V300A and observed the terminal voltage of the battery through the CAN BUS, and monitored the thermal chamber through and RS485. According to the measured current of a 96 s $2 p$ battery pack of a mid-size EV in test, the imported load current for a single cell was calculated before the bench experiment. The reference SOC, which is regarded as true SOC, is calculated by coulomb counting according to the known initial SOC and load current. The specifications of battery cell and the NBT BTS-5V300A are respectively shown in TABLE $\mathrm{V}$ and TABLE VI.

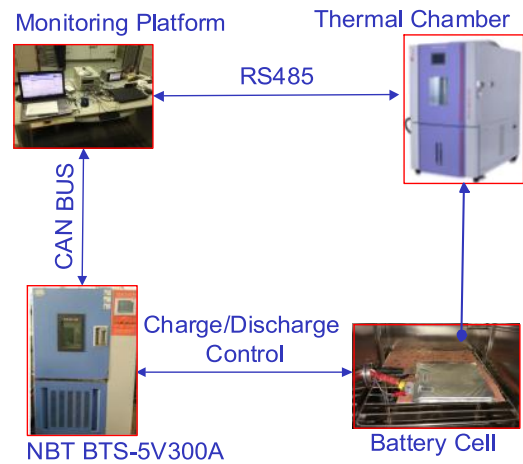

Fig. 4. Composition of battery test bench.

TABLE V

THE SPECIFICATIONS OF BATTERY CELL

\begin{tabular}{ll}
\hline \hline Items & Values \\
\hline Nominal capacity & $35000 \mathrm{mAh}$ \\
Rated voltage & $3700 \mathrm{mV}$ \\
maximum charging current of cell & $75 \mathrm{~A}$ \\
maximum discharging current of cell & $150 \mathrm{~A}$ \\
\hline \hline
\end{tabular}

TABLE VI

THE SPECIFICATIONS OF THE NBT BTS-5V300A

\begin{tabular}{ll}
\hline \hline Items & Values \\
\hline Voltage measurement range for charge & $10 \mathrm{mV} \sim 5 \mathrm{~V}$ \\
Voltage measurement range for discharge & $2 \mathrm{~V} \sim 5 \mathrm{~V}$ \\
Current measurement error & $0.1 \%$ of full scale \\
Voltage measurement error & $0.1 \%$ of full scale \\
Temperature resolution & $0.1{ }^{\circ} \mathrm{C}$ \\
Sample frequency & $10 \mathrm{~Hz}$ \\
\hline \hline
\end{tabular}

\section{B. Experimental Details}

\section{1) Experiment 1: Test of DST Cycles}

The dynamic stress test (DST) is a dynamic driving test profile simplified from actual urban driving cycles (AUDC), which is widely used to evaluate performance, control strategies and 
algorithmic effects of vehicles. In the DST experiment of this study, the battery went through 27 DST cycles and the battery SOC changed from the initial 1 to 0.2672 . The profiles of load

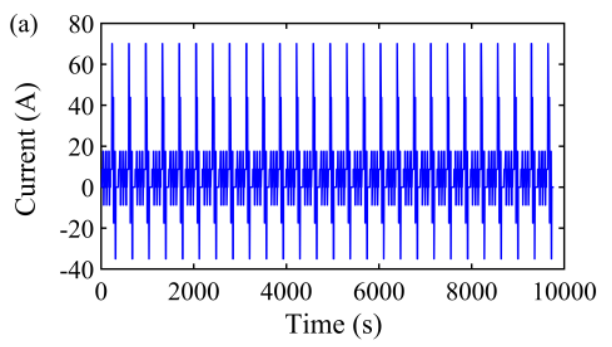

(a) current and terminal voltage in the entire test are shown in Fig. 5, in which the positive current values represent discharging current, and the negative current values represent charging current.

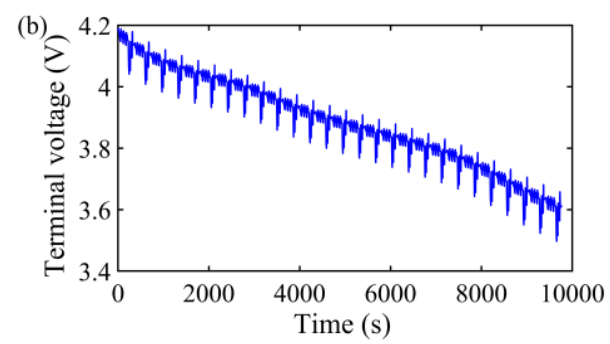

(b)

Fig. 5. Experimental profiles of DST:(a) Current of entire test; (b) Terminal voltage of entire test.

\section{2) Experiment 2: Test of UDDS Cycles}

The UDDS test is a relatively complex typical dynamic driving cycle, which is often used to evaluate vehicle performance, energy consumption and battery management

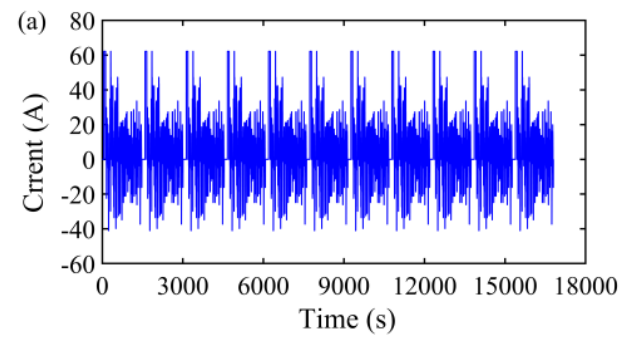

(a) strategy. In this study, 11 UDDS cycles were utilized to evaluate the SOC estimation method, and the battery SOC decreased from 1 to 0.1812 in the entire test. The profiles of load current and terminal voltage are shown in Fig. 6.

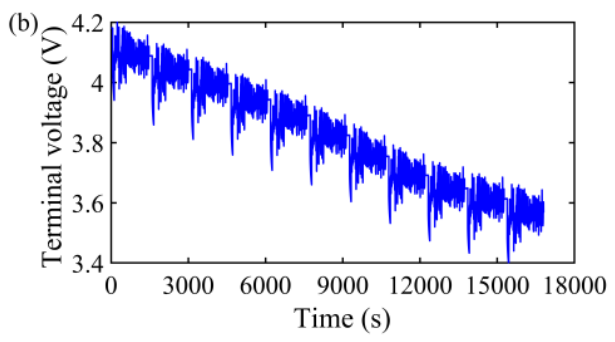

(b)

Fig. 6. Experimental profiles of UDDS:(a) Current of entire test; (b) Terminal voltage of entire test.

\section{3) Experiment 3: Test of DST Cycles with Measurement Noise}

Due to the measurement deviation of current and voltage sensors and the influence of external disturbance signals, SOC estimation faces great challenge. To evaluate the anti-disturbance ability of the proposed method, a test of DST cycles with measurement noise was conducted. In this experiment, a Gaussian white noise disturbance signal was added to the measurement of current and voltage under the same DST conditions as in section 4.4.1. (62) is the probability density function of the random noise in the single cycle. The standard deviation of the noise is calculated by (63):

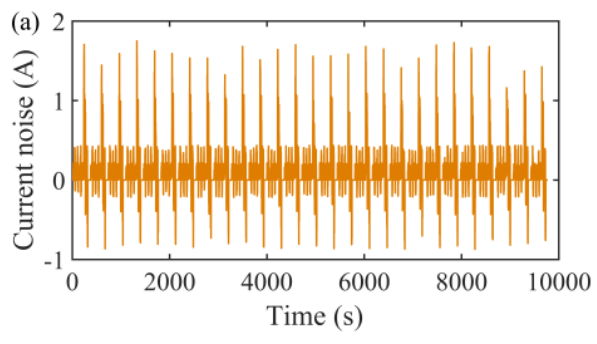

(a)

$$
\begin{aligned}
& f(x)=\frac{1}{\sqrt{2 \pi \sigma}} \exp \left(-\frac{x^{2}}{2 \sigma^{2}}\right) \\
& \sigma=\alpha N_{\max } / 3
\end{aligned}
$$

where $x$ represents current noise or terminal voltage noise, $\sigma$ is the standard deviation of the noise distribution, $\alpha$ is the proportion coefficient, $N_{\max }$ donates the maximum value of the current or the voltage. Considering the practical condition of electric vehicles, the proportion coefficient was set as $2.5 \%$. The current and terminal voltage noise profiles in this test are shown in Fig. 7.

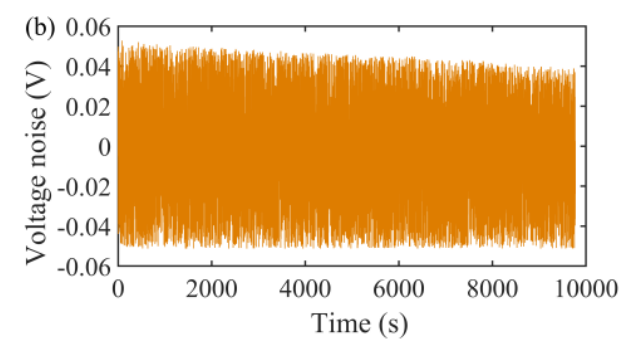

(b)

Fig. 7. Experimental profiles of noise for DST:(a) Current noise of entire test; (b) Terminal voltage noise of entire test. 


\section{Simulation result and Discussion}

The estimation simulation of DST was made in Matlab/Simulink. The online parameter identification profiles and the reference values are shown in Fig. 8. The reference values are offline obtained through HPPC test [51], while the identification profiles are obtained by using VRLS. Fig. 8 suggests that the $R_{0}$ values of identification and reference are close in low SOC region to high region. the identification values of $R_{p}$ and $C_{p}$ general approach to those of reference, but the deviations in high SOC region are relatively large. As stated in our research of [51], the influence of $R_{p}$ and $C_{p}$ to SOC estimation are much smaller than that of $R_{0}$. It can be expected that the identification effect is enough. The cause of the large error region will study in our later research.

The SOC estimation profiles of DST are exhibited in Fig. 9 and Fig. 10. In the verification tests, the initialized estimated SOC value is 0 while the corresponding one of reference is 1 . With intent to illustrate the performance of the proposed method, the estimation results using the proposed algorithm and the combination of RLS and SEKF (RLS+SEKF) are compared. The model parameters are identified with VRLS in both methods. The estimation results of RLS+SEKF and the proposed method are presented in Fig. 9 and Fig. 10, respectively. The estimated SOC of both methods converges quickly to the reference value in about $65 \mathrm{~s}$, because the same algorithm is used before filtering convergence. It is shown in Fig. 9 that the estimation accuracy of RLS+SEKF method is poor and has large error fluctuation. In comparison, it can be seen from Fig. 10 that the maximum absolute estimation error of the proposed method is less than 0.01 , and the errors are distributed in the region near zero after

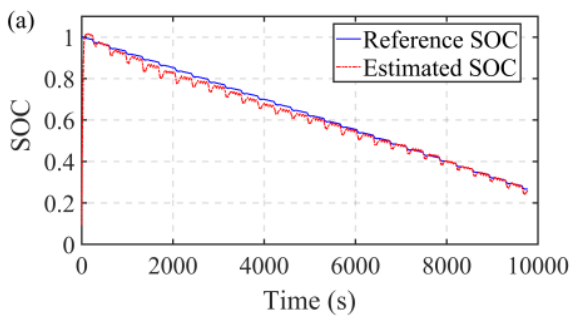

(a)

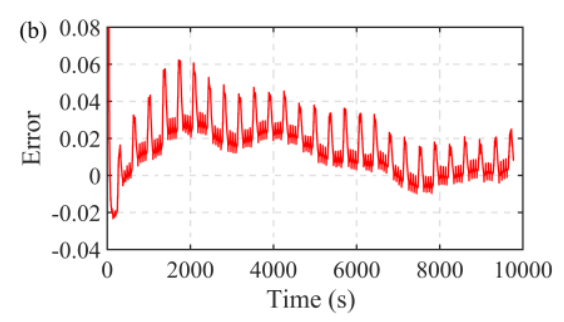

(b)

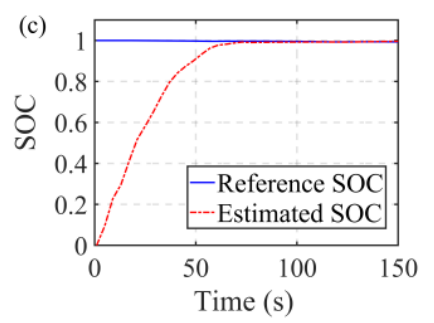

(c)

Fig. 9. Estimation result of RLS+SEKF in DST cycles. (a) Estimated SOC; (b) Estimation error; (c) Convergence detail. 


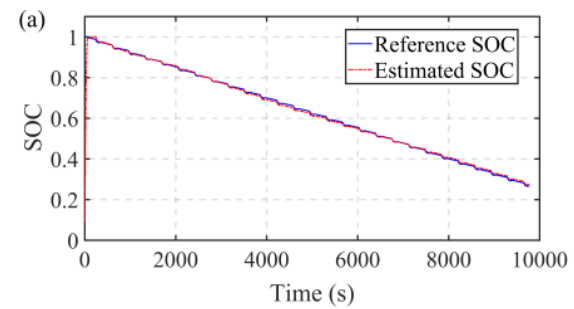

(a)

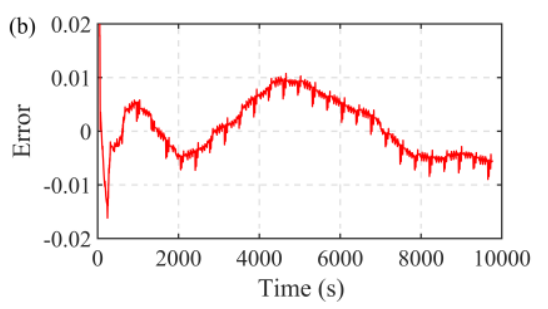

(b)

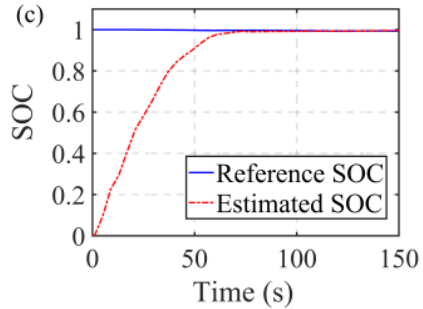

(c)

Fig. 10. Estimation result of proposed method in DST cycles. (a) Estimated SOC; (b) Estimation error(c) Convergence detail.

The SOC estimation profiles of UDDS are presented in Fig. 11 and Fig. 12. In the UDDS test, the estimated SOC of both methods converges to the reference value in about 60 s, because both methods used the same estimator before filtering convergence. Both RLS+SEKF method and the proposed method have high estimation accuracy during the early stage of the algorithm operation. However, as the algorithm continues, the fixed noise covariances $Q$ and $R$ are no longer applicable to the system, which leads to the error expansion of the SOC estimation of SEKF. The absolute estimation error band of the proposed method is within 0.015 , and most of the errors are less than 0.01 and distributed around 0 . The estimation result suggests that the proposed method can track system noise well and accurately estimate SOC in the entire process. The proposed method operated well in both DST and UDDS, which indicates its high SOC estimation accuracy and strong robustness.

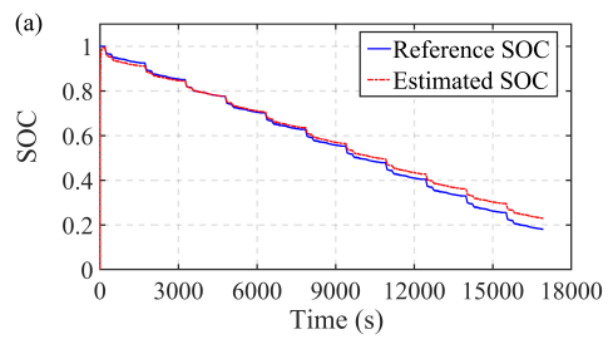

(a)

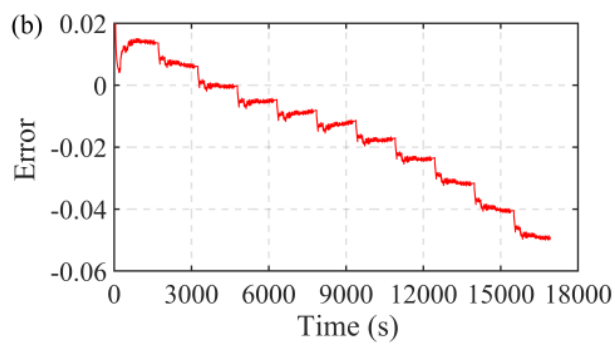

(b)

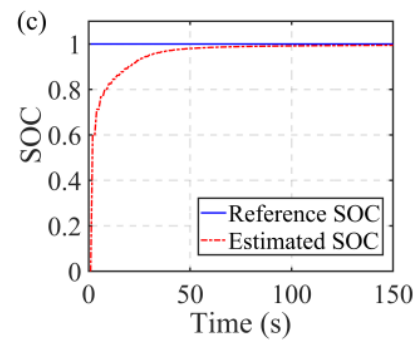

(c)

Fig. 11. Estimation result of RLS+SEKF in UDDS cycles; (a) Estimated SOC. (b) Estimation error; (c) Convergence detail.

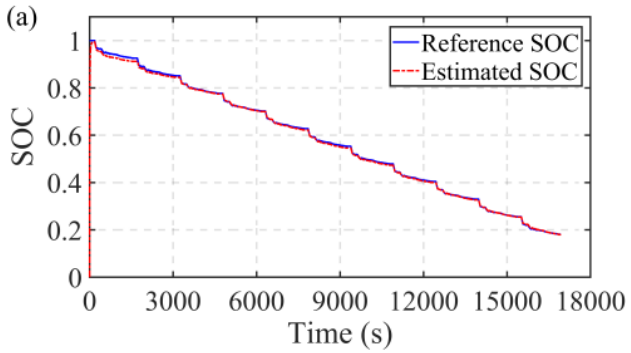

(a)

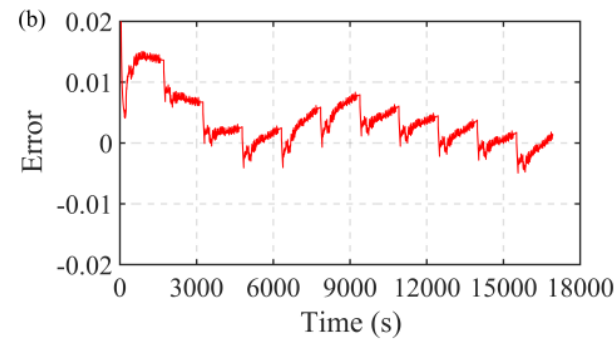

(b)

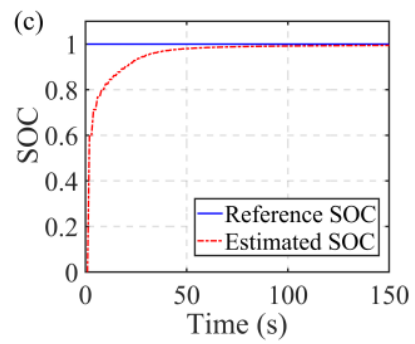

(c)

Fig. 12. Estimation result of proposed method in UDDS cycles. (a) Estimated SOC; (b) Estimation error; (c) Convergence detail.

The SOC estimation result profiles of DST cycles with measurement noise are presented in Fig. 13 and Fig. 14. In Fig. 13 , it is shown that the maximum absolute estimation error of $\mathrm{RLS}+\mathrm{SEKF}$ method is larger than 0.1 , and the dispersion degree is high. Comparing Fig. 13 and Fig. 14, it is seen that the estimation error of proposed method before using adaptive extended Kalman filter is as large as RLS+SEKF method. The estimator performs well after the new AEKF is started up, and the maximum absolute error of proposed method is less than 0.02. The result indicates that the new AEKF can reduce the disturbance of measurement bias of current and voltage. 


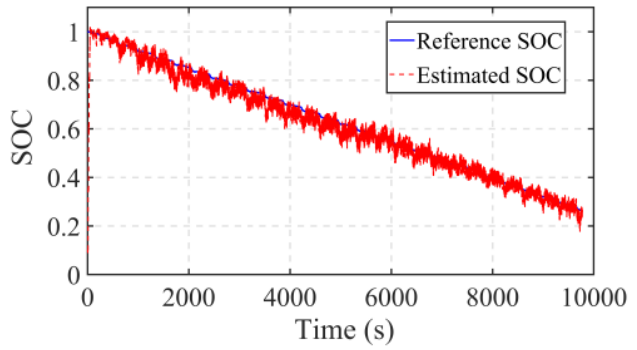

(a)

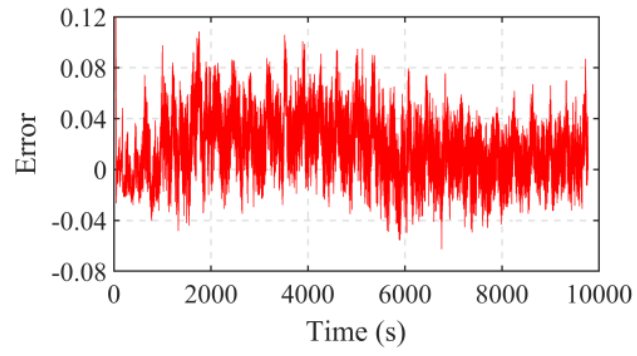

(b)

Fig. 13. Estimation result of RLS+SEKF in DST cycles with measurement noise. (a) Estimated SOC; (b) Estimation error.

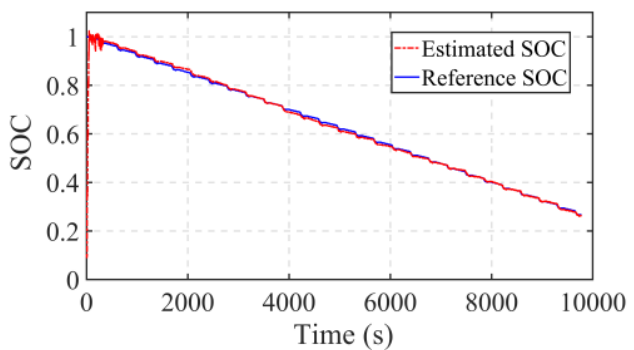

(a)

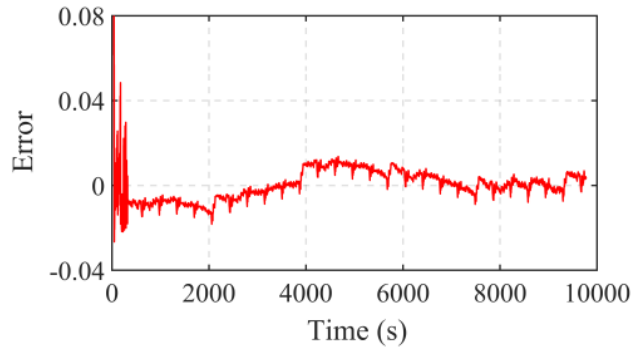

(b)

Fig. 14. Estimation result of proposed method in DST cycles with measurement noise. (a) Estimated SOC; (b) Estimation error.

Current offset is another common sensor error, which will cause the most error when coulomb counting because the error will be integrated. In order to verify the proposed method, a DST with current offset is created. The modified current measurement is calculated by (64).

$I_{\text {new }}=I_{\text {meas }}+I_{\text {offset }}$

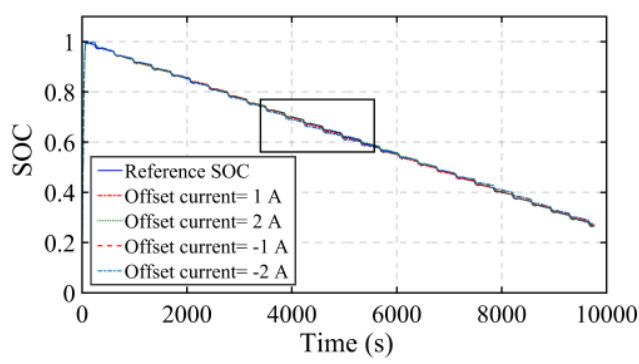

(a) where $I_{\text {meas }}$ is raw current measurement, $I_{\text {offset }}$ is the added current offset, and the used values of $I_{\text {offset }}$ are $1 \mathrm{~A}, 2 \mathrm{~A},-1 \mathrm{~A}$ and -2 A. The estimation result is presented in Fig. 15. The maximum estimation absolute error is less than 0.015 , suggesting the high robustness of the algorithm.

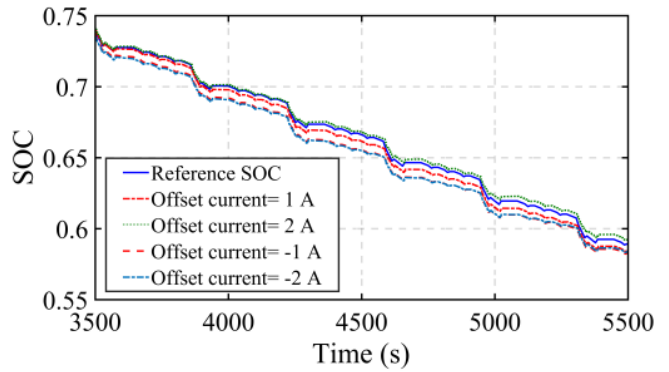

(b)

Fig. 15. Estimation result of proposed method in DST cycles with current offset. (a) Estimated SOC; (b) The local details.

The estimation accuracy of SEKF relies remarkably on the appropriate predetermination of the process noise covariance matrix $Q$ and measurement noise covariance matrix $R$. The use of inappropriate noise parameters will lead to large estimation error even filtering divergence. Overcoming this shortcoming is an ability that an adaptive filter should have. To further evaluate the robustness of the proposed method, several different combinations of $Q$ and $R$ were used in the two algorithms for estimation simulation of UDDS test. The values of different $Q$ and $R$ used for simulation are listed in TABLE VII. Where $I_{2 \times 2}$ is the identity matrix of 2 rows and 2 columns.

The simulation results are presented in Fig. 16 and Fig. 17. It 
can be seen from the results that with different initial noise parameters, the estimation accuracy of the proposed method is excellent, while the estimation accuracy of RLS+SEKF is heavily dependent on the predetermination of noise covariances. The result comparison indicates the great anti-interference capacity of the proposed method.

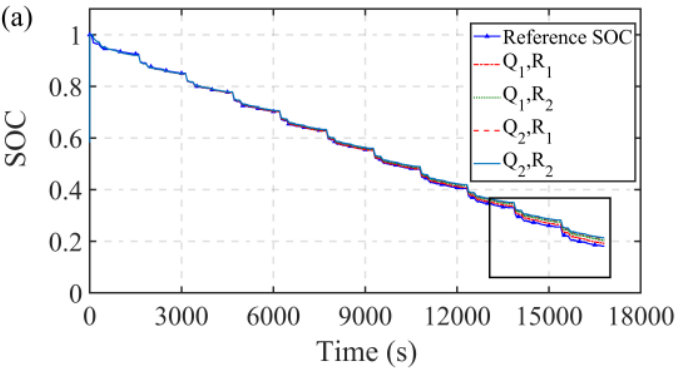

(a)
TABLE VII THE DIFFERENT Q AND R USED FOR SIMULATION

\begin{tabular}{ccc}
\hline \hline$J$ & $Q_{J}$ & $R_{J}$ \\
\hline 1 & $I_{2 \times 2} \times 10^{-7}$ & 0.5 \\
2 & $I_{2 \times 2} \times 10^{-8}$ & 1.5 \\
\hline \hline
\end{tabular}

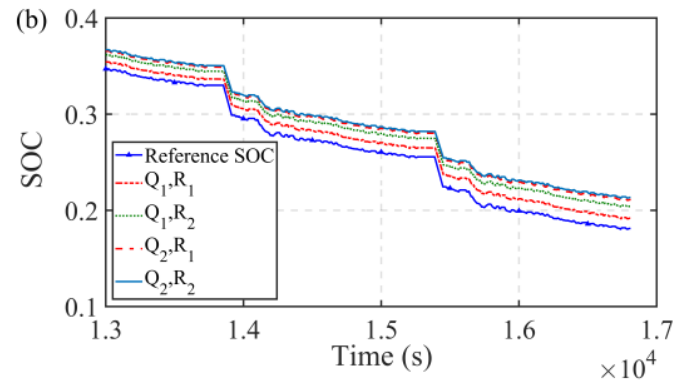

(b)

Fig. 16. Estimation result of RLS+SEKF with different noise parameters for UDDS. (a) Estimated SOC; (b) The local details.

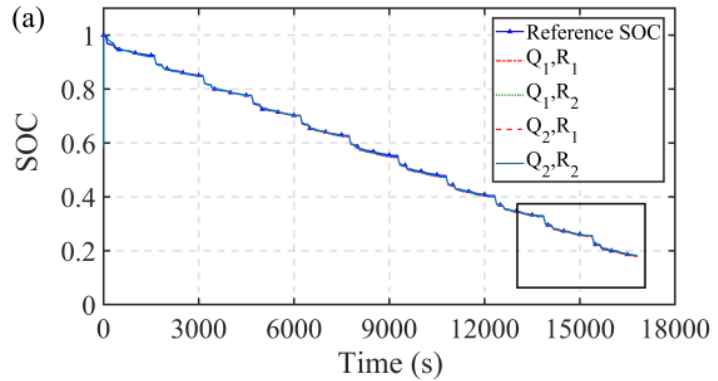

(a)

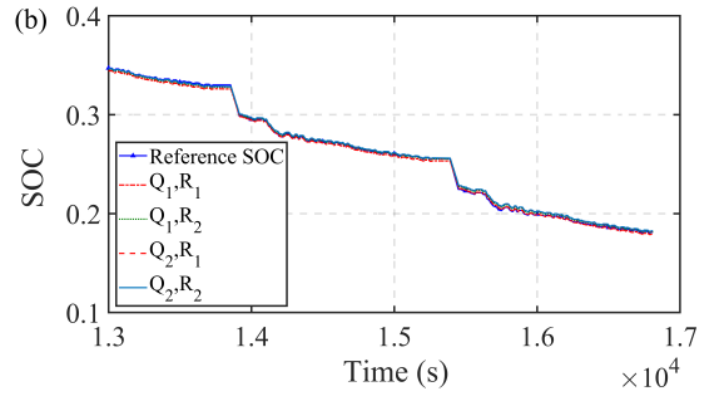

(b)

Fig. 17. Estimation result of proposed method with different noise parameters for UDDS. (a) Estimated SOC; (b) The local details.

\section{CONCLUSION}

Equivalent circuit model-based methods are suitable for SOC estimation of lithium-ion battery, and EKF is an appropriate SOC estimator. However, the lithium-ion battery system varies during its operation, and offline parameter identification approaches suffer from the difficulty of implementation. Furthermore, the system noise, which comes from the uncertainties of model and measurement, is unmeasurable and not known in advance, thus the predetermined fixed noise parameters will significantly affect the accuracy of the SOC estimation. In order to solve these problems, this paper proposes a SOC estimation method combining RLS and a novel adaptive extended Kalman filter, which can online dynamically track the model parameters and the system noise. RLS is used for online identification of the ECM, while the new adaptive extended Kalman filter tracks the system noise by directly adapting to priori error covariance $P_{k \mid k-1}$. Bench experiments and simulations were established to evaluate the proposed method, and the proposed method was compared to the RLS+SEKF method. The maximum estimation error after the convergence of the proposed method in DST and UDDS is less than 0.015 , which is far less than that of traditional RLS+SEKF. Further, a test of DST cycles with current and terminal voltage noise is conducted to evaluate the anti-disturbance ability of the proposed method. In the disturbance environment, the absolute estimation errors are limited within a region less than 0.02 after starting up the new adaptive extend Kalman filter. The experiments demonstrate the 
high estimation accuracy and strong robustness of the proposed method.

In the future study, the proposed method will be applied to BMS software development to verify its practicality in engineering.

\section{ACKNOWLEDGEMENTS}

The project is supported by National Key R\&D Program of China (2016YFB0100903-2), the Foundation for Innovative Research Groups of the National Natural Science Foundation of China (Grant No. 51621004) and the Natural Science Foundation of China (Grant No. U1864207), the Opening Project of the Guangxi Key Laboratory of Automobile Components and Vehicle Technology of Guangxi University of Science and Technology (No. 2017GKLACVTKF01).

\section{REFERENCES}

[1] W. Helmut, W. Thomas, Z. Herbert, "Large lithium-ion battery-powered electric vehicles - From idea to reality," IEEE Trans. Veh. Technol., vol. 42, pp. 4383-4394, 2018.

[2] S. Farhad, A. Nazari, "Introducing the energy efficiency map of lithium-ion batteries," Int J Energy Res, vol. 43, pp. 1-14, 2018.

[3] Y. Wu, W. Wang, J. Ming, et al., “An Exploration of New Energy Storage System: High Energy Density, High Safety, and Fast Charging Lithium Ion Battery," Adv Funct Mater, vol. 29, pp. 1805978, 2018.

[4] C. Zou, L. Zhang, X. Hu, Z. Wang, T. Wik, M. Pecht, "A review of fractional-order techniques applied to lithium-ion batteries, lead-acid batteries, and supercapacitors," J Power Sources, vol. 390, pp. 286-296, 2018.

[5] C. Hu, B. D. Youn, J. Chung, "A multiscale framework with extended Kalman filter for lithium-ion battery SOC and capacity estimation," Appl Energy, vol. 92, pp. 694-704, 2012.

[6] M-H. Hung, C-H. Lin, L-C. Lee, C-M. Wang, "State-of-charge and state-of-health estimation for lithium-ion batteries based on dynamic impedance technique," J Power Sources, vol. 268, pp. 861-873, 2014.

[7] C. Unterrieder, C. Zhang, M. Lunglmayr, R. Priewasser, S. Marsili, M. Huemer, "Battery state-of-charge estimation using approximate least squares," J Power Sources, vol. 278, pp. 274-286, 2015.

[8] L. Zhao, M. Lin, Y. Chen, "Least-squares based coulomb counting method and its application for state-of-charge (SOC) estimation in electric vehicles," Int J Energy Res, vol. 40, pp. 1389-1399, 2016.

[9] B. Pattipati, B. Balasingam, GV. Avvari, KR. Pattipati, Y. Bar-Shalom, "Open circuit voltage characterization of lithium-ion batteries," J Power Sources, vol. 269, pp. 317-333, 2014.

[10] AJ. Salkind, C. Fennie, P. Singh, T. Atwater, DE. Reisner, "Determination of state-of-charge and state-of-health of batteries by fuzzy logic methodology," J Power Sources, vol. 80, pp. 293-300, 1999.
[11] A. Affanni, A. Bellini, C. Concari, G. Franceschini, E. Lorenzani, C. Tassoni, "EV battery state of charge: neural network based estimation," Electric Mach Drives Conf, 2003 IEMDC'03 IEEE Int, vol. 2, pp. 684- 688, 2003.

[12] IS. Kim, "Nonlinear State of Charge Estimator for Hybrid Electric Vehicle Battery," IEEE Transactions on Power Electronics, vol. 23, pp. 2027-2034, 2008.

[13] B. Xia, C. Chen, Y. Tian, W. Sun, Z. Xu, W. Zheng, "A novel method for state of charge estimation of lithium-ion batteries using a nonlinear observer," J Power Sources, vol. 270, pp. 359-366, 2014.

[14] JN. Hu, JJ. Hu, HB. Lin, XP. Li, CL. Jiang, XH. Qiu, WS. $\mathrm{Li}$, "State-of-charge estimation for battery management system using optimized support vector machine for regression,” J Power Sources, vol. 269, pp. 682-693, 2014.

[15] J. Xu, C. Mi, B. Cao, J. Deng, "The State of Charge Estimation of Lithium-Ion Batteries Based on a Proportional-Integral Observer," IEEE Trans. Veh. Technol., vol. 63, pp. 1614-1621, 2014.

[16] H. Chaoui, C. Christopher Ibe-Ekeocha, "State of Charge and State of Health Estimation for Lithium Batteries Using Recurrent Neural Networks," IEEE Trans. Veh. Technol., vol. 66, pp. 8773-8783, 2017.

[17] L. Kang, X. Zhao, J. Ma, “A new neural network model for the state-of-charge estimation in the battery degradation process," Appl Energy, vol. 121, pp. 20-27. 2014.

[18] X. Hu, H. Yuan, C. Zou, Z. Li, L. Zhang, "Co-Estimation of State of Charge and State of Health for Lithium-Ion Batteries Based on Fractional-Order Calculus," IEEE Trans. Veh. Technol., vol. 67, pp. 10319-10329, 2018.

[19] S. Li, M. Hu, Y. Li, C. Gong, "Fractional-order modeling and SOC estimation of lithium-ion battery considering capacity loss," Int J Energy Res, vol. 43, pp. 417-429, 2019.

[20] S. Sepasi, R. Ghorbani, BY. Liaw, "Inline state of health estimation of lithium-ion batteries using state of charge calculation,” J Power Sources, vol. 299, pp. 246-254. 2015.

[21] Z. Xi, M. Dahmardeh, B. Xia, Y. Fu, C. Mi, "Learning of Battery Model Bias for Effective State of Charge Estimation of Lithium-Ion Batteries," IEEE Trans. Veh. Technol., vol. 68, pp. 8613-8628, 2019.

[22] G. Fan, X. Li, M. Canova, "A Reduced-Order Electrochemical Model of Li-Ion Batteries for Control and Estimation Applications," IEEE Trans. Veh. Technol., vol. 67, pp. 76-91, 2018.

[23] E. Prada, D. Di Domenico, Y. Creff, J. Bernard, V. Sauvant-Moynot, F. Huet, "Simplified electrochemical and thermal model of LiFePO4-graphite Li-ion batteries for fast charge applications," J Electrochem Soc, vol. 159, pp. A1508-1519, 2012.

[24] GL. Plett, "Extended Kalman filtering for battery management systems of LiPB-based HEV battery packs: Part 1, Background," J Power Sources, vol. 134, pp. 252-261, 2004.

[25] GL. Plett, "Extended Kalman filtering for battery management systems of LiPB-based HEV battery packs: Part 2, Modeling and identification," J Power Sources, vol. 134, pp. 262-276, 2004.

[26] GL. Plett, "Extended Kalman filtering for battery 
management systems of LiPB-based HEV battery packs: Part 3, State and parameter estimation," J Power Sources, vol. 134, pp. 277-292, 2004.

[27] T. Wang, S. Chen, H. Ren, Y. Zhao, "Model-based unscented Kalman filter observer design for lithium-ion battery state of charge estimation," Int J Energy Res, vol. 42, pp. 1603-1614, 2018.

[28] J. Peng, J. Luo, H. He, B. Lu, "An improved state of charge estimation method based on cubature Kalman filter for lithium-ion batteries," Appl Energy, vol. 253, pp. 113520, 2019.

[29] X. Cui, Z. He, E. Li, A. Cheng, M. Luo, Y. Guo, "State-of-charge estimation of power lithium-ion batteries based on an embedded micro control unit using a square root cubature Kalman filter at various ambient temperatures," Int J Energy Res, vol. 43, pp. 3561-3577, 2019.

[30] X. Cui, Z. Jing, M. Luo, Y. Guo, H. Qiao, "A new method for state of charge estimation of lithium-ion batteries using square root cubature Kalman filter," Energies, vol. 11, pp. 209, 2018.

[31] T. Wada, T. Takegami, Y. Wang, "Sequential estimation of state of charge and equivalent circuit parameters for lithium-ion batteries," 2015 American Control Conference (ACC), Chicago, IL, pp. 2494-2498, 2015.

[32] Y. Li, J. Chen, F. Lan, "Enhanced online model identification and state of charge estimation for lithium-ion battery under noise corrupted measurements by bias compensation recursive least squares," Journal of Power Sources, Vol. 456, 227984, ISSN 0378-7753, 2020.

[33] Z. Wei, J. Zhao, D. Ji, KJ. Tseng, "A multi-timescale estimator for battery state of charge and capacity dual estimation based on an online identified model," Appl Energy, vol. 204, pp. 1264-1274, 2017.

[34] D. Sun, X. Chen, "Adaptive parameter identification method and state of charge estimation of Lithium Ion battery," 2014 17th International Conference on Electrical Machines and Systems (ICEMS), Hangzhou, pp. 855-860, 2014.

[35] Y. Qiu, X. Li, W. Chen, Z. Duan, L. Yu, "State of charge estimation of vanadium redox battery based on improved extended Kalman filter," ISA Transactions, Vol. 94, Pages 326-337, 2019.

[36] L. Zhong, C. Zhang, Y. He, Z. Chen, "A method for the estimation of the battery pack state of charge based on in-pack cells uniformity analysis," Appl Energy, vol. 113, pp. 558-564, 2013.

[37] F. Sun, R. Xiong, "A novel dual-scale cell state-of-charge estimation approach for series-connected battery pack used in electric vehicles," J Power Sources, vol. 274, pp. 582-594, 2015.

[38] F. Sun, R. Xiong, H. He, "A systematic state-of-charge estimation framework for multi-cell battery pack in electric vehicles using bias correction technique," Appl Energy, 162: 1399-1409, 2016.

[39] M. Dubarry, N. Vuillaume, BY. Liaw, "From single cell model to battery pack simulation for Li-ion batteries," J Power Sources, vol. 186, pp. 500-507, 2009.

[40] M. Dubarry, BY. Liaw, "Development of a universal modeling tool for rechargeable lithium batteries," J Power Sources, vol. 174, pp. 856-860, 2007.

[41] D. Andre, M. Meiler, K. Steiner, H. Walz, T. Soczka-Guth, DU. Sauer, "Characterization of high-power lithium-ion batteries by electrochemical impedance spectroscopy. II: Modelling," J Power Sources, vol. 196, pp. 5349-5356, 2011.

[42] Y. Zheng, M. Ouyang, X. Han, L. Lu, J. Li, "Investigating the error sources of the online state of charge estimation methods for lithium-ion batteries in electric vehicles," J Power Sources, vol. 377, pp. 161-188, 2018.

[43] RK. Mehra, "Approaches to adaptive filtering," IEEE Transactions on Automatic Control, vol. 17, pp. 693-698, 1972.

[44] X. Zhang, C. Wang, L. Gao and X. Fan, "Prediction of State of Charge of Lithium Battery for Electric Vehicle Based on Data Driven and Model Fusion," 2018 11th International Symposium on Computational Intelligence and Design (ISCID), Hangzhou, China, pp. 161-164., 2018.

[45] R. Xiong, H. He, F. Sun, K. Zhao, "Evaluation on state of charge estimation of batteries with adaptive extended Kalman filter by experiment approach,” IEEE Trans. Veh. Technol.", vol. 62, pp. 108-117, 2013.

[46] R. Xiong, F. Sun, H. He, TD. Nguyen, "A data-driven adaptive state of charge and power capability joint estimator of lithium-ion polymer battery used in electric vehicles," Energy, vol. 63, pp. 195-308, 2013.

[47] Y. Xu, M. Hu, A. Zhou, Y. Li, S. Li, C. Fu, C. Gong, "State of charge estimation for lithium-ion batteries based on adaptive dual Kalman filter," Applied Mathematical Modelling, Vol. 77, pp. 1255-1272, 2020.

[48] CK. Chui, G. Chen, "Kalman Filtering with Real-Time Applications,” Springer, Berlin Heidelberg, 2017.

[49] AH. Mohamed, KP. Schwarz, "Adaptive Kalman filtering for INS/GPS,” J Geodesy, vol. 73, pp. 193-203, 1999.

[50] J. Wang, J. Wang, D. Zhang, X. Shao, G. Chen, "Kalman filtering through the feedback adaption of prior error covariance," Signal Processing, vol. 152, pp. 47-53, 2018.

[51] X. Cui, Z. He, E. Li, A. Cheng, M. Luo, Y. Guo, "state-of-charge estimation of power lithium-ion batteries based on an embedded micro control unit using a square root cubature Kalman filter at various ambient 
temperatures," International Journal of Energy Research, vol. 43, pp. 3561-3577, 2019. 